Article

\title{
Underwater Laser Treatment of PET: Effect of Processing Parameters on Surface Morphology and Chemistry
}

\author{
Jakub Siegel $^{1, *}$, Pavla Šuláková ${ }^{1}$, Markéta Kaimlová ${ }^{1}$, Václav Švorčík ${ }^{1}$ and Tomáš Hubáček ${ }^{2}$ \\ 1 Department of Solid State Engineering, University of Chemistry and Technology Prague, \\ 16628 Prague, Czech Republic; pavla.sulakova@vscht.cz (P.Š.); polivkoa@vscht.cz (M.K.); \\ vacla.svorcik@vscht.cz (V.Š.) \\ 2 SoWa Research Infrastructure, Biology Center of the Czech Academy of Sciences, \\ 37005 České Budějovice, Czech Republic; hubacektom@gmail.com \\ * Correspondence: jakub.siegel@vscht.cz; Tel.: +420-22-044-5149
}

Received: 1 November 2018; Accepted: 23 November 2018; Published: 26 November 2018

\begin{abstract}
Rapid development of nanotechnology in processes of metal nanoparticle immobilization on solid surfaces, especially polymeric ones, requires the study of particular issues within these complex approaches. Numerous studies have been published on laser light mediated manipulation with single metal nanoparticles in water environment and even laser assisted immobilization of such particles on polymeric substrate, however, not much has been reported on fundamentals of underwater laser processing of polymer itself, especially regarding to resulting surface morphology and chemistry. In this work, we study surface morphology (atomic force microscopy (AFM)) and chemistry (angle-resolved X-ray photoelectron spectroscopy (ARXPS) and inductively coupled plasma-mass spectroscopy (ICP-MS)) of polyethylene terephthalate (PET) after underwater laser treatment in broad scale of applied laser fluencies and operating voltages. Due to typical dependence of laser efficiency on operating voltage, induced nanostructures on PET exhibited a noticeable symmetry spread out around the maxima of laser efficiency for low laser fluencies. The study of surface chemistry revealed that at high laser fluencies, photochemical decomposition of macromolecular polymer structure took place, resulting in rapid material ablation and in balanced chemical composition of the surface throughout the studied profile. Enrichment of the water bath by the low-molecular polymer degradation products proves that ablation mechanism is the governing process of surface nanostructure formation in underwater laser processing.
\end{abstract}

Keywords: polyethylene terephthalate; excimer laser; underwater treatment; surface morphology; surface chemistry

\section{Introduction}

With the development of new approaches of nanoparticle immobilization on polymer surfaces, especially those driven by forward-directed scattering forces in colloid solutions [1], the need to thoroughly investigate laser to polymer interaction in a water environment becomes of fundamental importance. Many theoretical models have been suggested to describe the processes at the water-polymer interface during laser treatment, however, experimental studies, addressing this issue in detail, are either missing or date back to early 1990s, which hinders comparison of obtained data to modern analytical outputs of present science. Several models of laser heating of polymers in air have been suggested so far. These include photothermal models [2,3], especially for higher wavelength radiation (such as for infrared lasers). Contrary to that, in case of UV lasers, models based 
on both photothermal and photodissociative or photochemical breakdown have been reported $[4,5]$. The photochemical model fundamentals lie in the chemical kinetics of bond dissociation and are coupled with the processes of photothermal decomposition of material. Some of these models also take into account dynamic attenuation of radiation by the expanding plasma plume [6]. Besides these, molecular dynamics is also frequently used to simulate laser ablation of polymers [7]. Although the fundamentals of the laser-matter interaction remain the same as in air even in the presence of water, such environment can have a significant effect especially to the heating of the polymer and in turn may result to the development of surface structures, which morphology is far away from those, typically observed in case of laser processed materials in air environment (LIPSS) [8,9]. In particular, the phenomena of plasma formation, its expansion, and subsequent radiation attenuation effects, are greatly influenced by the specific type of confining media [10].

In this work, we report on investigation of surface morphology and chemistry of polyethylene terephthalate (PET) after underwater treatment with excimer $\mathrm{KrF}$ laser in broad scale of applied laser fluencies $\left(4-20 \mathrm{~mJ} \cdot \mathrm{cm}^{-2}\right)$ and operating voltages (19-29 kV). Due to typical parabolic dependence of laser efficiency on operating voltage of main laser capacitor, induced structures exhibit a noticeable symmetry spread out around the maxima of laser efficiency at lower examined laser fluencies. Development of typical surface nanostructure takes place in a narrow interval of applied laser fluencies and is accompanied by considerable polymer ablation as the laser fluence increases.

\section{Materials and Methods}

\subsection{Materials, Apparatus and Procedures}

Polyethylene terephthalate foil (PET, thickness $50 \mu \mathrm{m}$, density $1.36 \mathrm{~g} \cdot \mathrm{cm}^{-3}$ ) supplied by Goodfellow Ltd., Cambridge, UK, was exposed to a 248-nm KrF excimer laser (COMPexPro 50F, Coherent, Inc., California, USA) with pulse duration of 20 to $40 \mathrm{~ns}$, frequency of $10 \mathrm{~Hz}, 6000$ pulses. A laser beam was linearly polarized by fused quartz prism (model PBSO 248-100) and directed through the aperture of $5 \times 10 \mathrm{~mm}^{2}$ to the polymer. Strip of PET foil $\left(30 \times 8 \mathrm{~mm}^{2}\right)$ was placed vertically into high precision spectro-optics cell (HellmaAnalitics GmbH, Müllheim, Germany, type No. 100-QS, light path of $10 \mathrm{~mm}$ ), so that the polymer was centered in the middle of the cuvette. Afterwards, $3 \mathrm{~mL}$ of water (water for ion chromatography, Sigma-Aldrich Co., Missouri, USA) was added by using automatic pipette. Scheme of the process in depicted in Figure 1. In our experiments, we applied fluencies ranging from 4 to $20 \mathrm{~mJ} \cdot \mathrm{cm}^{-2}$, however, worm-like morphology, typical for underwater polymer processing with linearly polarized laser light, occurred in much narrower interval of 8 to $18 \mathrm{~mJ} \cdot \mathrm{cm}^{-2}$. All PET samples were modified under static conditions with 6000 pulses, perpendicularly to polymer surface. For the setting of required laser fluence $\left(4-20 \mathrm{~mJ} \cdot \mathrm{cm}^{-2}\right)$ at different operating voltage (19-29 kV, charging voltage applied on main storage capacitor), external laser beam attenuator (Attenuator module No. 2910216, Coherent, Inc., California, USA) was used. Attenuator was placed immediately behind the mouth of the laser tube.

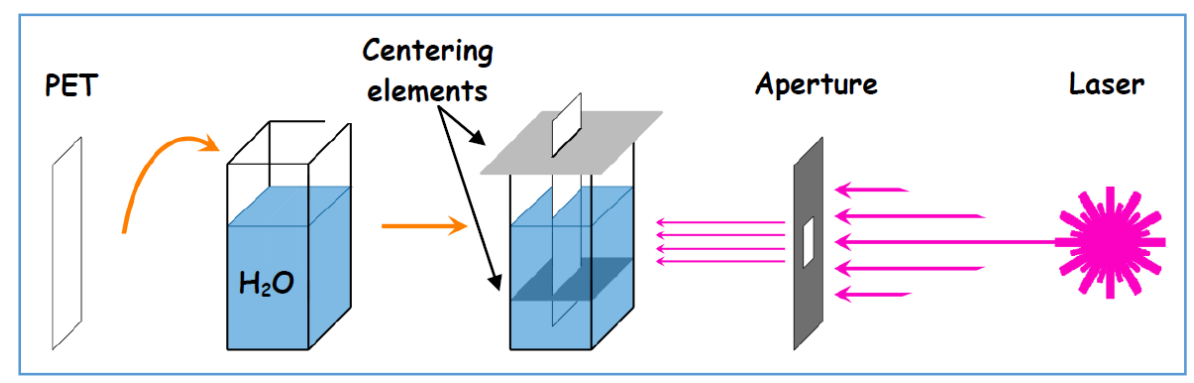

Figure 1. Scheme of underwater laser processing of polyethylene terephthalate (PET) showing key process steps. 


\subsection{Analytical Methods}

Laser fluence, applied on PET during underwater treatment, was measured by FieldMaxII-TOP Laser Power Meter (Coherent, Inc., California, USA), so that it was placed just behind the cuvette-filled water without the polymer. Owing to only negligible absorption of purified water at $248 \mathrm{~nm}$ (absorption coefficient $\varepsilon_{\mathrm{H} 2 \mathrm{O}}<0.04$ [11]), measured laser fluence in that way corresponds well to that applied to polymer during the experiments.

The atomic concentrations of elements, carbon $\mathrm{C}(1 \mathrm{~s})$ and oxygen $\mathrm{O}(1 \mathrm{~s})$, were studied by Angle Resolved X-ray Photoelectron Spectroscopy (ARXPS) on Omicron Nanotechnology ESCAProbeP spectrometer, Taunusstein-Neuhof, Germany. The X-ray source was monochromated at $1486.7 \mathrm{eV}$. The evaluation of spectra was performed by CasaXPS software, version 2.3.19. The samples were studied under the electron take-off angles of 0 and $81^{\circ}$.

Surface morphology and roughness were examined by atomic force microscope (AFM) VEECO CP II device in a tapping mode. A Veeco oxide-sharpened P-doped silicon probe RTESPA-CP was used. Surface roughness, characterized by the mean roughness value $\left(R_{\mathrm{a}}\right)$, represents the arithmetic average of the deviation from the center plane of a sample. Variation of $R_{\mathrm{a}}$ parameter did not exceed $5 \%$. All samples were measured at 7 different points within modified area in the arrangement 2, 3, 2 along the longer side of the modified region (see Figure 2). In all cases the measurement provided similar surface morphologies within one sample, pointing to homogeneous distribution of induced nanostructure by laser treatment. Presented AFM images were carefully chosen to represent typical morphologies of laser processed samples under the specific modification conditions.

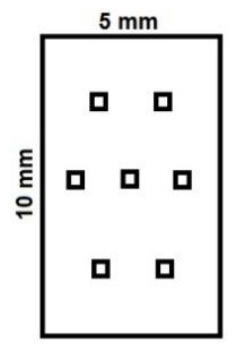

Figure 2. Distribution of measuring points within laser treated area during atomic force microscopy (AFM) analysis.

Inductively coupled plasma with mass spectroscopy detector (ICP-MS) was used to determine total carbon content (TCC) in water from the cuvette after laser treatment of polymer. Changes in the TCC correspond to the release of low molecular weight polymer ablation product to water environment due to laser modification. The trace element analysis of TCC was conducted by using Agilent 8800 triple-quadrupole spectrometer (Agilent Technologies, California, USA) connected to an auto-sampler according to measurement protocol taken from [12]. Sample nebulization was performed using a MicroMist device equipped with a tempered cell. To minimize the interference of an analyte with multiatomic adducts, we used a collision cell (He collision gas) operating in a high-energy mode with double quadrupole filtration.

\section{Results and Discussion}

\subsection{Surface Morphology}

Development of typical surface morphology during underwater laser treatment of PET was observed in narrow interval of laser fluencies from 8 to $18 \mathrm{~mJ} \cdot \mathrm{cm}^{-2}$, regardless of operating voltage applied on laser main storage capacitor. Operating voltage, however, had distinctive impact on the fine microstructure superimposed on the on PET surface, in particular at low applied fluencies. This interesting phenomenon most likely originates from the symmetry of laser efficiency on operating 
voltage (see Figure 3), which reaches a maximum at about $25 \mathrm{kV}$ (ca 1\%) and then falls evenly to both border values (ca $0.75 \%$ ).

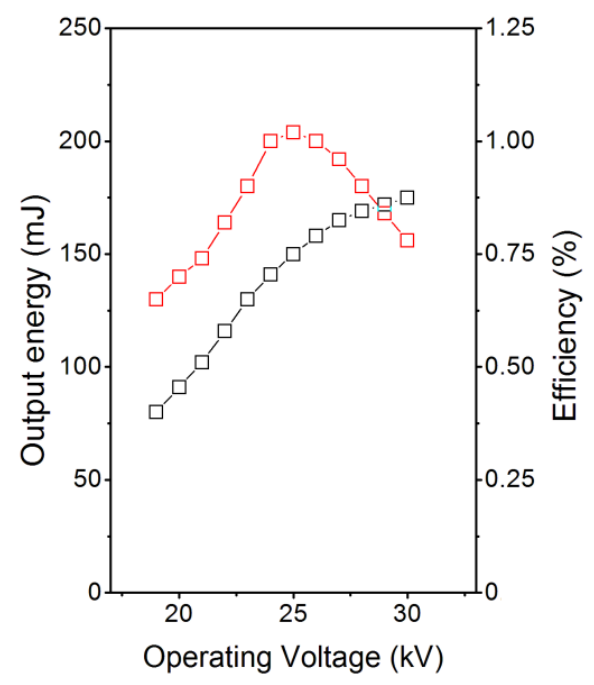

Figure 3. Dependence of output energy (black) and laser efficiency (red) on charging voltage of laser main storage capacitor of $\mathrm{KrF}$ laser.

Lower laser efficiency at low and/or high voltages is related to increased instability in laser beam profile at both border values [13], which obviously determines the resulting surface morphology, especially at lower laser fluencies. For clear illustration of this phenomenon, surface morphology in dependence of operating voltages (19-29 kV) is shown in Figures 4-6 for applied laser fluencies of 8,12 , and $16 \mathrm{~mJ} \cdot \mathrm{cm}^{-2}$, respectively. We have chosen these fluencies, since they provide sufficient insight into the nature of morphology changes of underwater laser processed polymer. With increasing flow, massive ablation of the polymer surface took place, until the typical worm-like nanostructure disappeared completely (applied laser fluence $>18 \mathrm{~mJ} \cdot \mathrm{cm}^{-2}$ ).

One can clearly see that at lower laser fluencies and boarder values of applied voltages (Figure 4), the developed surface morphology is reminiscent to that of treated in air [14]. Even though the LIPSS are not precisely developed, typical prolongation of worm-like structures at specific operating voltage into linear ones is obvious. This may originate from the confined plasma plume in water, which expands at a much lower velocity than in air (order of magnitude less). This implicates that spatially confined plasma induces increase of surface temperature in water environment much intensively than in air [10], which may significantly contributes to the development of similar surface structure as in air when processing at sufficiently lower laser fluencies in water [15]. It is also obvious from the Figures 4-6, that the higher the laser fluence applied the more uniform developed nanostructure is and the possibility of modulation of fine microstructure by variation of applied voltage diminishes. This finding may be quantified by corresponding values of surface roughness, which are given in Table 1. Concurrently to AFM images, $R_{\mathrm{a}}$ values suggest that at higher applied fluencies the effect of voltage on surface roughness is negligible and surface roughness stabilizes, regardless of specific voltage value. At low fluence, when worm-like nanostructure is not fully developed (see Figure 4: 19 and $29 \mathrm{kV}$ ), however, the variation of $R_{\mathrm{a}}$ value corresponds to the possibility to vary surface morphology just by proper setting of applied voltage. 

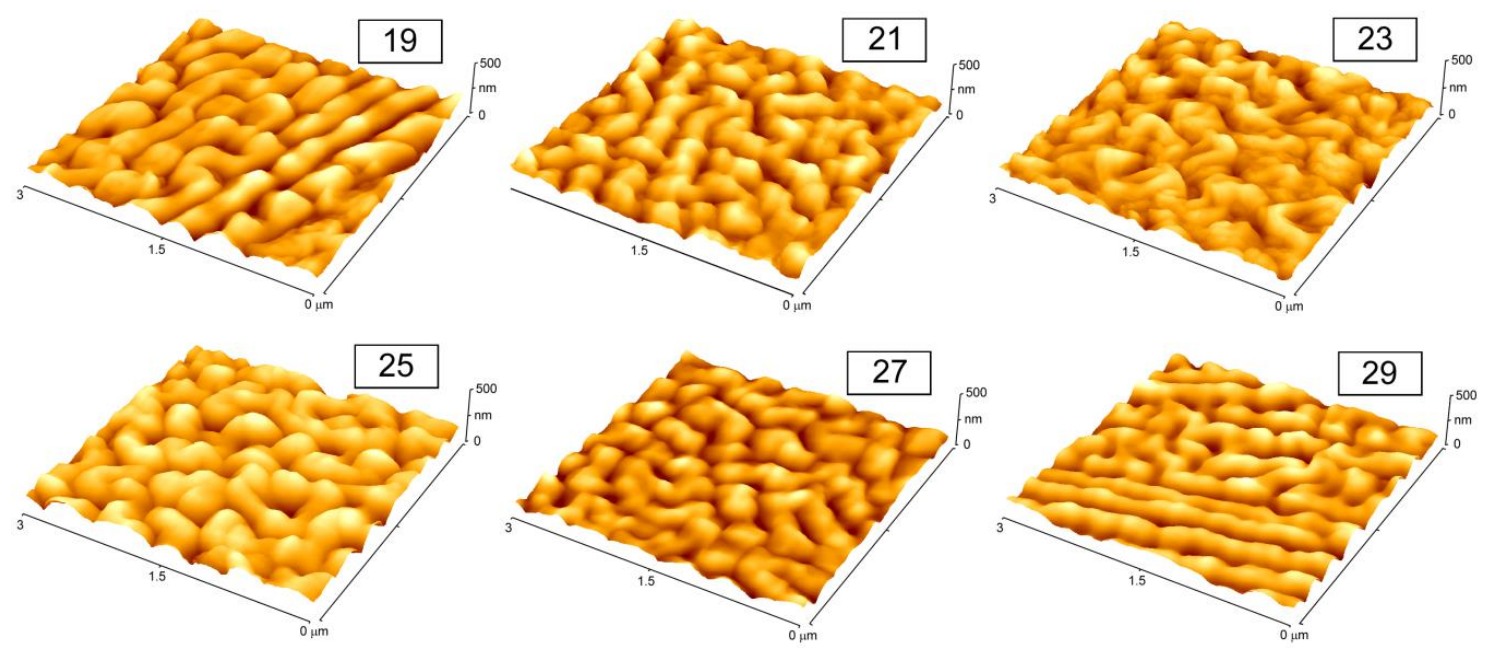

Figure 4. AFM images of underwater laser treated PET at laser fluence of $8 \mathrm{~mJ} \cdot \mathrm{cm}^{-2}$ and different values of operating voltages. Numbers in frames refer to laser operating voltages in $\mathrm{kV}$.
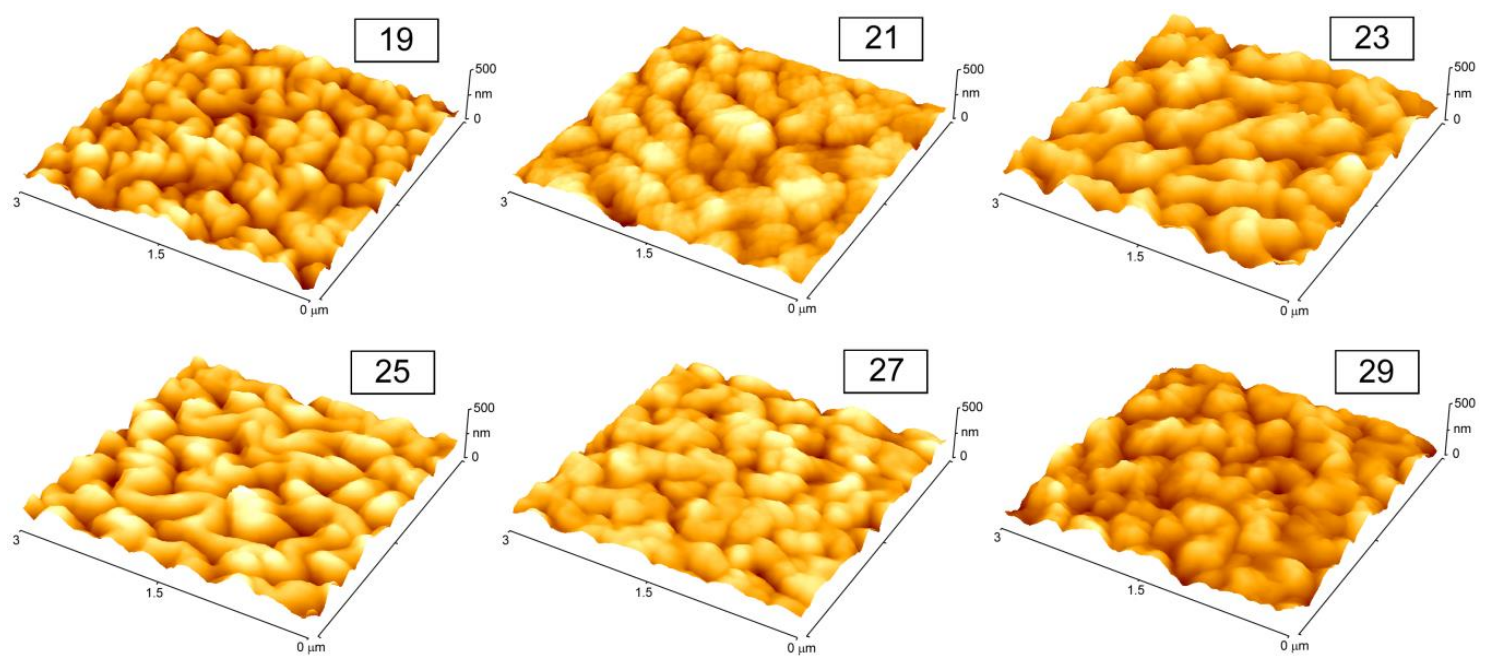

Figure 5. AFM images of underwater laser treated PET at laser fluence of $12 \mathrm{~mJ} \cdot \mathrm{cm}^{-2}$ and different values of operating voltages. Numbers in frames refer to laser operating voltages in $\mathrm{kV}$.
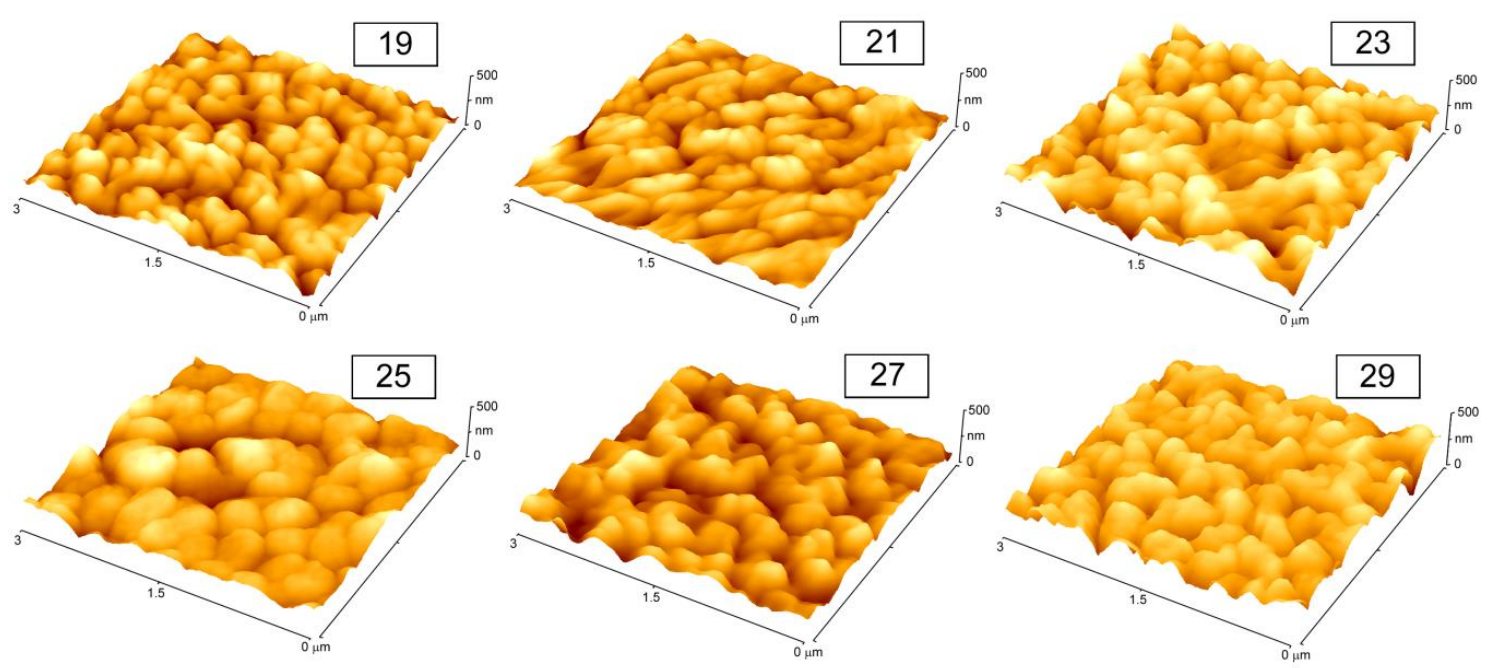

Figure 6. AFM images of underwater laser treated PET at laser fluence of $16 \mathrm{~mJ} \cdot \mathrm{cm}^{-2}$ and different values of operating voltages. Numbers in frames refer to laser operating voltages in $\mathrm{kV}$. 
Table 1. Surface roughness $R_{\mathrm{a}}$ (in $\mathrm{nm}$ ) of underwater laser treated samples at laser fluencies of $0,8,12$, and $16 \mathrm{~mJ} \cdot \mathrm{cm}^{-2}$ and different values of applied voltages. Presented $R_{\mathrm{a}}$ stands for the average value from seven measurements performed on each sample (according to Figure 2); variation of $R_{\mathrm{a}}$ value did not exceed $5 \%$.

\begin{tabular}{cccccccc}
\hline \multirow{2}{*}{ Laser Fluence $\left[\mathbf{m J} \cdot \mathbf{c m}^{-\mathbf{2}}\right]$} & \multicolumn{7}{c}{ Applied Voltage $[\mathbf{k V}]$} \\
\cline { 2 - 8 } & $\mathbf{0}$ & $\mathbf{1 9}$ & $\mathbf{2 1}$ & $\mathbf{2 3}$ & $\mathbf{2 5}$ & $\mathbf{2 7}$ & $\mathbf{2 9}$ \\
\hline $\mathbf{0}$ & 0.7 & - & - & - & - & - & - \\
$\mathbf{8}$ & - & 25.2 & 23.4 & 22.6 & 21.8 & 23.3 & 25.3 \\
$\mathbf{1 2}$ & - & 28.3 & 28.7 & 31.6 & 30.3 & 28.8 & 29.0 \\
$\mathbf{1 6}$ & - & 31.2 & 30.6 & 33.6 & 32.3 & 31.9 & 31.8 \\
\hline
\end{tabular}

\subsection{Chemical Composition of the Surface and Water Bath}

Changes in chemical composition of polymer surface after underwater laser treatment were recorded by ARXPS and are given in Table 2. Atomic concentrations of $\mathrm{O}$ (35.2 at. \%) and $\mathrm{C}$ $(64.8$ at. \%) in pristine PET corresponds well to polymer stoichiometry with theoretical concentrations of $\mathrm{O}(33.0$ at. \%) and C (67.0 at. \%), which are often shifted in favor of carbon due to hydrocarbon impurities being adsorbed on the surfaces of the polar polymeric materials from air [16]. Advantage of angle-resolved XPS analysis lies in the possibility to sensitively distinguish between the element concentration in thick surface polymer layer (typically 10 atomic layers, electron collection angle of $0^{\circ}$ ) and ultrathin surface shell (typically 1 to 2 atomic layers, electron collection angle of $81^{\circ}$ ). Thus, samples of pristine PET typically exhibit increase of carbon concentration when analyzed under $81^{\circ}$, especially due to absorbed hydrocarbons in mono-atomic layer (see Table 2).

Table 2. Atomic concentrations of $\mathrm{C}(1 \mathrm{~s})$ and $\mathrm{O}(1 \mathrm{~s})$ in pristine and underwater treated PET measured by ARXPS under electron take-off angles of 0 and $81^{\circ}$ for different operating voltages.

\begin{tabular}{|c|c|c|c|c|}
\hline \multirow[t]{2}{*}{ Laser Fluence $\left[\mathrm{mJ} \cdot \mathrm{cm}^{-2}\right]$} & \multirow{2}{*}{$\begin{array}{c}\text { Angle } \\
{\left[{ }^{\circ}\right]}\end{array}$} & \multirow{2}{*}{$\begin{array}{c}\text { Voltage } \\
{[\mathrm{kV}]}\end{array}$} & \multicolumn{2}{|c|}{$\begin{array}{c}\text { Concentration } \\
\text { [at. \%] }\end{array}$} \\
\hline & & & $\mathrm{C}$ & $\mathbf{O}$ \\
\hline \multirow{2}{*}{$\mathbf{0}$} & 0 & \multirow{2}{*}{0} & 35.2 & 64.8 \\
\hline & 81 & & 49.8 & 50.2 \\
\hline \multirow{4}{*}{8} & 0 & \multirow{2}{*}{19} & 75.5 & 24.5 \\
\hline & 81 & & 83.3 & 16.7 \\
\hline & 0 & \multirow{2}{*}{25} & 76.3 & 23.7 \\
\hline & 81 & & 81.5 & 18.5 \\
\hline \multirow{4}{*}{12} & 0 & \multirow{2}{*}{19} & 79.7 & 20.3 \\
\hline & 81 & & 83.8 & 16.2 \\
\hline & 0 & \multirow{2}{*}{25} & 79.6 & 20.4 \\
\hline & 81 & & 82.3 & 17.7 \\
\hline \multirow{4}{*}{16} & 0 & \multirow{2}{*}{19} & 81.2 & 18.8 \\
\hline & 81 & & 82.0 & 18.0 \\
\hline & 0 & \multirow{2}{*}{25} & 80.9 & 19.1 \\
\hline & 81 & & 82.1 & 17.9 \\
\hline
\end{tabular}

Apparently, when increasing applied laser fluence the discrepancy between "bulk" and "very surface" concentration of $\mathrm{C}$ and $\mathrm{O}$ gradually disappears. This phenomenon most likely originates from (i) massive polymer ablation (absorbed hydrocarbons are removed), (ii) dramatic photochemical decomposition of macromolecular structure which unifies the chemical composition of the surface in a deeper profile [4]. Both of these contributions being affected by considerable changes in surface morphology, especially regarding data obtained under $81^{\circ}$, where the analytical information 
comes predominantly from the ridges of induced nanostructure. Besides this, these changes are obviously accompanied by considerable graphitization of polymer surface [1] which contributes to increasing concentration of carbon with increasing applied fluence. This finding is similar to that of Elaboudi et al. [15], who identified the hydrolysis reaction responsible for decrease of laser threshold during excimer laser processing of polyesters which increases the efficiency at low applied fluencies. Their results showed that ablation products are rich in carboxylic groups and carbon, but did not correspond directly to hydrolysis products of polymer. It is also evident, that variation of voltage has no direct impact on chemical composition, which corresponds well with the fact that absorbed energy is invariant towards this parameter. Remarkable difference in concentrations of $\mathrm{C}$ and $\mathrm{O}$ in dependence of detection angle can only be observed at treatment fluence of $8 \mathrm{~mJ} \cdot \mathrm{cm}^{-2}$, similarly as in case of pristine PET. This phenomenon most likely originates from considerably low absorbed energy when the ablation and photothermal cleavage of macromolecules is low.

To reveal the mechanism of surface structure development on laser treated polymer, identification of the rate of material ablation was accomplished. To address this issue, ICP-MS analysis of the water bath used in underwater laser treatment, aiming to total carbon content (TCC), was accomplished. Those results are summarized in Figure 7a,b. Changes in the TCC correspond to the release of low molecular weight polymer ablation product to water environment due to laser modification. Figure $7 \mathrm{a}$ shows dependence of TCC on applied voltage at fixed laser fluence of $8 \mathrm{~mJ} \cdot \mathrm{cm}^{-2}$. Compared to measured TCC background in fresh water (blue line, $1.2 \pm 0.3 \mathrm{mg} \cdot \mathrm{L}^{-1}$ ), TCC in the bath increased approximately one order of magnitude after laser processing. It is clearly visible that applied voltage has no influence on TCC, which is in good concordance with results obtained from AR-XPS analysis, suggesting limited material removal from the PET surface at low fluencies.

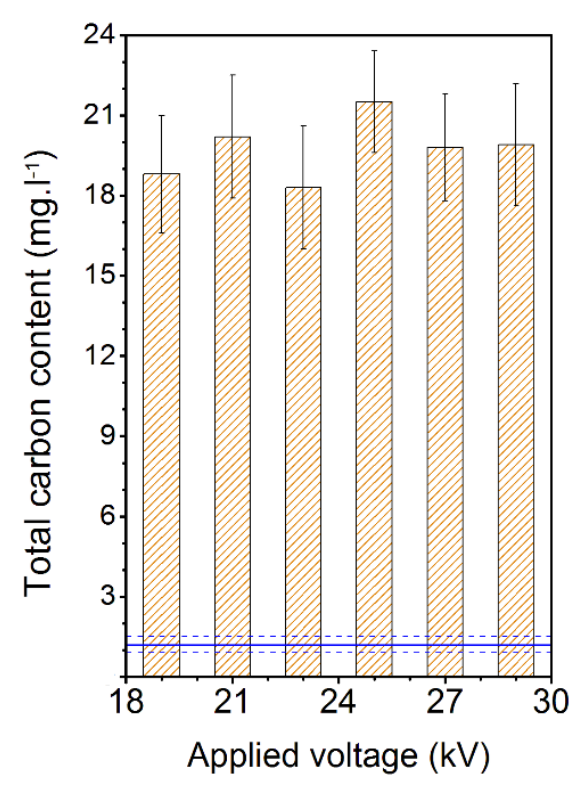

(a)

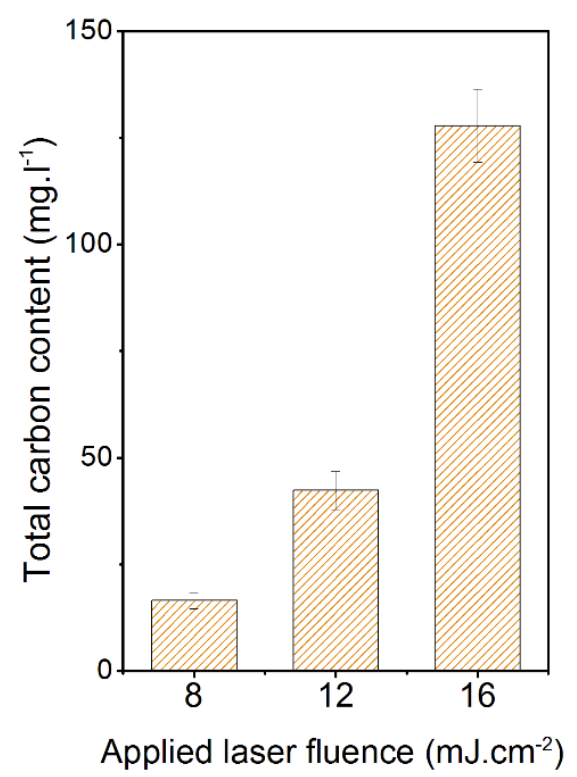

(b)

Figure 7. Total carbon content (TCC) determined by ICP-MS in water bath after laser processing of PET: (a) TCC in dependence of applied voltage at fixed value of laser fluence of $8 \mathrm{~mJ} \cdot \mathrm{cm}^{-2}$ and (b) TCC independence of applied laser fluence at fixed value of applied voltage of $25 \mathrm{kV}$. Blue line (solid one) in (a) represents background TCC content in pure water together with measurement error (dash one).

Additionally to that, Figure $7 \mathrm{~b}$ summarizes the values of TCC measured for different laser fluence applied at fixed voltage of $25 \mathrm{kV}$. From the presented data, one can see exponential dependence of the amount of ablated material on applied fluence. As the absorbed energy grows, increase of TCC is detected, pointing to massive polymer ablation. Data presented in Figures 5 and 6 unambiguously demonstrate the enrichment of the water bath by the low-molecular polymer degradation products, 
which prove that ablation mechanism is the governing process of surface nanostructure formation. Besides others, this finding contributed to reveal one of the challenges of present material research, namely the mechanism of LIPPS formation. Great efforts have been made to address this issue so far, considering various approaches involving UV-Vis spectrometry [17] or gravimetry [18], which outputs were often loaded with considerable statistic error. Right the use of water bath and thus capturing of low molecular weight product of ablation process into the media allowed to determine TCC by ICP-MS method.

\section{Conclusions}

In this work we studied surface morphology and chemistry of polyethylene terephthalate after underwater laser processing in broad scale of applied laser fluencies and operating voltages. We determined the interval of laser fluencies causing development of typical "worm-like" structure on PET. While the value of laser fluence was crucial with respect to the development of specific nanostructure, the effect of operating voltage lied especially in gentle modulation of primary "worm-like" nanostructure at considerably low laser fluencies. Most reproducible structures were prepared at operating voltages closed to the maxima of laser efficiency, where the beam profile exhibited considerable stability. Increase of laser fluence significantly supported photochemical decomposition of polymer macromolecules, resulting in massive material removal which unified the chemical composition of the surface in entire accessible depth of angle resolved XPS analysis. Data from ICP-MS unambiguously proved that ablation mechanism is the governing process of surface nanostructure formation in the process of underwater laser processing of PET.

Author Contributions: J.S. designed the experiments, supervised the study, and wrote the manuscript. P.Š. and M.K. realized underwater laser experiments and performed AFM analysis. T.H. performed and evaluated ICP-MS analysis. V.Š. contributed to evaluation of analyses outputs and reviewed the final manuscript.

Funding: This research was funded by Czech Science Foundation, grant number 18-07619S.

Acknowledgments: The authors hereby thank Adéla Siegelová for personal assistance in the realization of the research.

Conflicts of Interest: The authors declare no conflicts of interest.

\section{References}

1. Siegel, J.; Lyutakov, O.; Polívková, M.; Staszek, M.; Hubáček, T.; Švorčík, V. Laser-assisted immobilization of colloid silver nanoparticles on polyethyleneterephthalate. Appl. Surf. Sci. 2017, 420, 661-668. [CrossRef]

2. Cain, S.R. A photothermal model for polymer ablation-Chemical modification. J. Phys. Chem. 1993, 97, 7572-7577. [CrossRef]

3. Bityurin, N.; Malyshev, A. Bulk photothermal model for laser ablation of polymers by nanosecond and subpicosecond pulses. J. Appl. Phys. 2002, 92, 605-613. [CrossRef]

4. Sadoqi, M.; Kumar, S.; Yamada, Y. Photochemical and photothermal model for pulsed-laser ablation. J. Thermophys. Heat Transf. 2002, 16, 193-199. [CrossRef]

5. Heitz, J.; Reisinger, B.; Fahrner, M.; Romanin, C.; Siegel, J.; Svorcik, L. Laser-induced periodic surface structures (LIPSS) on polymer surfaces. In Proceedings of the 14th International Conference on Transparent Optical Networks (ICTON 2012), Coventry, UK, 2-5 July 2012; IEEE: New York, NY, USA, 2012. [CrossRef]

6. Schmidt, H.; Ihlemann, J.; Wolff-Rottke, B.; Luther, K.; Troe, J. Ultraviolet laser ablation of polymers: Spot size, pulse duration, and plume attenuation effects explained. J. Appl. Phys. 1998, 83, 5458-5468. [CrossRef]

7. Conforti, P.F.; Prasad, M.; Garrison, B.J. Elucidating the thermal, chemical, and mechanical mechanisms of ultraviolet ablation in poly(methyl methacrylate) via molecular dynamics simulations. Acc. Chem. Res. 2008, 41, 915-924. [CrossRef] [PubMed]

8. Slepicka, P.; Siegel, J.; Lyutakov, O.; Kasalkova, N.S.; Kolska, Z.; Bacakova, L.; Svorcik, V. Polymer nanostructures for bioapplications induced by laser treatment. Biotechnol. Adv. 2018, 36, 839-855. [CrossRef] [PubMed] 
9. Krajcar, R.; Siegel, J.; Lyutakov, O.; Slepicka, P.; Svorcik, V. Optical response of anisotropic silver nanostructures on polarized light. Mater. Lett. 2014, 137, 72-74. [CrossRef]

10. Marla, D.; Zhang, Y.; Hattel, J.H.; Spangenberg, J. Modeling of nanosecond pulsed laser processing of polymers in air and water. Modell. Simul. Mater. Sci. Eng. 2018, 26. [CrossRef]

11. Dibble, W.E.; Kaszyk, J.; Tiller, W.A. Bulk water with exclusion zone water characteristics: Experimental evidence of interaction with a non-physical agent. Water 2014, 6, 35-44.

12. Nischwitz, V.; Gottselig, N.; Missong, A.; Meyn, T.; Klumpp, E. Field flow fractionation online with ICP-MS as novel approach for the quantification of fine particulate carbon in stream water samples and soil extracts. J. Anal. At. Spectrom. 2016, 31, 1858-1868. [CrossRef]

13. Bauerle, D. Laser Processing and Chemistry, 4th ed.; Springer: Berlin/Heidelberg, Germany, 2011; pp. 13-62, ISBN 978-3-642-17612-8.

14. Slepicka, P.; Nedela, O.; Siegel, J.; Krajcar, R.; Kolska, Z.; Svorcik, V. Ripple polystyrene nano-pattern induced by KRF laser. Express Polym. Lett. 2014, 8, 459-466. [CrossRef]

15. Elaboudi, I.; Lazare, S.; Belin, C.; Talaga, D.; Labrugère, C. Underwater excimer laser ablation of polymers. Appl. Phys. A 2008, 92, 743-748. [CrossRef]

16. Lewis, K.B.; Ratner, B.D. Observation of surface rearrangement of polymers using ESCA. J. Colloid Interface Sci. 1993, 159, 77-85. [CrossRef]

17. Barb, R.A.; Hrelescu, C.; Dong, L.; Heitz, J.; Siegel, J.; Slepicka, P.; Vosmanska, V.; Svorcik, V.; Magnus, B.; Marksteiner, R.; et al. Laser-induced periodic surface structures on polymers for formation of gold nanowires and activation of human cells. Appl. Phys. A 2014, 117, 295-300. [CrossRef]

18. Reznickova, A.; Chaloupka, A.; Heitz, J.; Kolska, Z.; Svorcik, V. Surface properties of polymers treated with F-2 laser. Surf. Interface Anal. 2012, 44, 296-300. [CrossRef]

(C) 2018 by the authors. Licensee MDPI, Basel, Switzerland. This article is an open access article distributed under the terms and conditions of the Creative Commons Attribution (CC BY) license (http://creativecommons.org/licenses/by/4.0/). 